\title{
Assistência ao pré-natal de baixo risco: avaliação da qualidade das consultas de enfermagem
}

\author{
Low-risk prenatal care: quality assessment of nursing consultations \\ Atención prenatal de bajo riesgo: evaluación de la calidad de las consultas de enfermería
}

Recebido: 05/11/2021 | Revisado: 12/11/2021 | Aceito: 16/11/2021 | Publicado: 26/11/2021

\author{
Suelane Coelho de Lima \\ ORCID: https://orcid.org/0000-0002-4547-6817 \\ Universidade Ceuma, Brasil \\ E-mail: coelhosuelane80@gmail.com \\ Patrícia dos Santos Silva Queiroz \\ ORCID: https://orcid.org/0000-0002-9587-1786 \\ Universidade Ceuma, Brasil \\ E-mail: patriciasqueiroz@gmail.com \\ Artur de Souza Veras \\ ORCID: https://orcid.org/0000-0002-6160-8736 \\ Universidade Estadual do Pará, Brasil \\ E-mail: lasferro1@gmail.com \\ Jullys Allan Guimarães Gama \\ ORCID: https://orcid.org/0000-0002-8834-6326 \\ Universidade Federal do Maranhão, Brasil \\ E-mail: jullys.gama@gmail.com \\ Francisco Alves Lima Júnior \\ ORCID: https://orcid.org/0000-0002-3117-4949 \\ Universidade Ceuma, Brasil \\ E-mail: francisco.enfdotrabalho@gmail.com \\ Érika Ferreira Tourinho \\ ORCID: https://orcid.org/0000-0003-2167-930X \\ Universidade Ceuma, Brasil \\ E-mail: tourinhoerika@gmail.com
}

\begin{abstract}
Resumo
Os profissionais de enfermagem desenvolvem um papel fundamental durante toda a gestação de uma mulher, tal como funções essenciais durante a assistência ao pré-natal. Devendo realizar tais ações de maneira eficaz, resguardando toda e qualquer necessidade biopsicossocial. Objetivo: Avaliar a assistência pré-natal desenvolvidas pela equipe de enfermagem no município de Campestre do Maranhão. Método: Estudo quantitativo, descritivo, realizado nas Unidades Básicas de Saúde, com 4 enfermeiras que realizam assistência ao pré-natal de baixo risco. O instrumento de coleta de dados foi através de um questionário desenvolvido pela autora. A coleta de dados ocorreu no mês de agosto de 2021. Os dados foram analisados em planilhas de Excel e através do google forms. Resultado: Ficou claro que o pré-natal de baixo risco no município de Campestre do Maranhão é realizado de forma satisfatória, apesar disso, há necessidades de as Unidades Básicas de Saúde melhorar o atendimento as gestantes. Conclusões: É possível observar uma qualidade satisfatória nas consultas de enfermagem preconizadas pelo caderno de atenção ao pré-natal de baixo risco. Nota-se que seja necessário qualificação profissional e educação permanente com o objetivo de melhorar o máximo a assistência.
\end{abstract}

Palavras-chave: Cuidado ao pré-natal; Assistência; Controle de qualidade.

\begin{abstract}
Nursing professionals play a key role throughout a woman's pregnancy, such as essential functions during prenatal care. They must perform such actions effectively, safeguarding any and all biopsychosocial needs. Objective: To evaluate the prenatal care developed by the nursing team in the city of Campestre do Maranhão. Method: Quantitative, descriptive study, carried out in Unidades Básicas de Saúde (Basic Health Units), with 4 nurses who provide low-risk prenatal care. The data collection instrument was through a questionnaire developed by the author. Data collection occurred in August 2021. The data was analyzed in Excel spreadsheets and through google forms. Result: It was clear that low-risk prenatal care in the municipality of Campestre do Maranhão is performed satisfactorily, despite this, there is a need for Basic Health Units to improve care for pregnant women. Conclusions: It is possible to observe a satisfactory quality in the nursing consultations recommended by the booklet for low-risk prenatal care. Professional qualification and continuing education are needed in order to improve care as much as possible.
\end{abstract}

Keywords: Prenatal care; Assistance; Quality control. 


\begin{abstract}
Resumen
Los profesionales de la enfermería desempeñan un papel fundamental durante todo el embarazo de la mujer, así como funciones esenciales durante los cuidados prenatales. Estas acciones deben llevarse a cabo de forma eficaz, salvaguardando todas y cada una de las necesidades biopsicosociales. Objetivo: Evaluar el cuidado prenatal desarrollado por el equipo de enfermería en el municipio de Campestre do Maranhão. Método: Estudio cuantitativo, descriptivo, realizado en Unidades Básicas de Salud, con 4 enfermeras que prestan asistencia al prenatal de bajo riesgo. El instrumento de recogida de datos fue un cuestionario elaborado por el autor. La recogida de datos se realizó en el mes de agosto de 2021. Los datos se analizaron en hojas de cálculo de Excel y mediante formularios de Google. Resultados: Está claro que el prenatal de bajo riesgo en el municipio de Campestre do Maranhão se realiza de forma satisfactoria, a pesar de ello, hay necesidades de las Unidades Básicas de Salud para mejorar la atención a las gestantes. Conclusiones: Se observa una calidad satisfactoria en las consultas de enfermería recomendadas por la cartilla para el control prenatal de bajo riesgo. Se señala que la cualificación profesional y la formación continua son necesarias para mejorar la asistencia en la medida de lo posible.
\end{abstract}

Palabras clave: Atención prenatal; Asistencia; Control de calidad.

\title{
1. Introdução
}

A criação de políticas de saúde com foco na saúde das mulheres contribuiu muito para a prevenção de doenças e agravos na vida de muitas mulheres. Graças a essas políticas, podemos ter hoje os cuidados durante a gestação, cuidados estes que serão voltados tanto para a saúde desta mulher como para o seu bebe, com estes cuidados podemos ter a chance de reduzir as taxas de morbidade e mortalidade materna e infantil. Um pré-natal de qualidade deve atenta-se a atender as reais necessidades das gestantes.

Quando a mulher descobre sua gestação, ela precisa compreender as mudanças que irão acontecer com o seu corpo. As adaptações no organismo precisam ser acompanhadas por um profissional pré-natalista, ainda no primeiro trimestre. No entanto, caso ela não apresente patologias, a gestação será considerada de baixo risco, sendo assim o enfermeiro e o médico, os profissionais mais atuantes na assistência prestada às gestantes (Valente et al., 2013).

O Programa de Humanização no Pré-natal e Nascimento (PHPN) fundamenta-se nos preceitos de que a humanização da assistência obstétrica e neonatal é condição primeira para o adequado acompanhamento do parto e puerpério (Brasil, 2002). Ao utilizar este termo, o ministério da saúde tinha como objetivo, melhorar as condições do atendimento através de mudanças nas assistências prestadas por profissionais que assistem à mulher gravida, de modo que este atendimento à mulher possa participar, incluindo a participação da família durante a gestação, parto e puerpério. De modo que este pré-natal deixe de ser centrado apenas no útero gravídico.

Os profissionais de enfermagem desempenham funções fundamentais na consulta da gestante no pré-natal, tais como, a orientação quanto a importância da consulta, exames necessários na gestação. Nesse sentindo, o enfermeiro deve realizar ações de maneira eficaz, resguardando a gestante de negligências, imperícias e imprudências, atuando de forma ética e responsável, para assegurar o nascimento da criança saudável (Lemes, 2012).

A assistência pré-natal, para ter qualidade, não precisa de procedimentos complexos e alta tecnologia, pois, um dos grandes segredos da qualidade desta assistência está na construção de um relacionamento de confiança entre os profissionais, a gestante e a sua família (Barbosa, 2007).

\section{Metodologia}

Este estudo trata-se de uma pesquisa exploratória de carácter descritiva com perspectiva quantitativa. De acordo com Gil (2017) a pesquisa descritiva busca levantar opiniões, atitudes e crenças de uma população. Esse tipo de pesquisa, visa descrever uma amostra. Foi conduzido nas unidades básicas de saúde de um município do interior do Maranhão. As unidades incluídas neste estudo foram: UBS Mãe Zefinha, UBS Alfredo Neres e UBS Justino Teixeira de Miranda da cidade de Campestre do Maranhão, no estado do Maranhão. 
O município de Campestre do Maranhão está situado na região Nordeste do país, no estado do Maranhão, constituída por aproximadamente 14. 530 habitantes (IBGE, 2021). Utiliza o sistema municipal de saúde de Atenção básica, composta por 6 unidades físicas, com 7 equipes com cobertura para área urbana e rural. A população alvo fora os profissionais de enfermagem que realizam o pré-natal de baixo risco durante suas consultas. Portanto, a amostra foi do tipo intencional com a participação de 4 enfermeiras que participam das UBS na região urbana.

O critério de inclusão foi ser enfermeiro nas UBS do município, realizar o pré-natal de baixo risco durante suas consultas de enfermagem e ter assinado o Termo de Consentimento Livre e Esclarecido. E como critério de exclusão, os profissionais que não faz parte da equipe da UBS.

O instrumento de coleta de dados, foi desenvolvido pela pesquisadora, contendo questões abertas e fechadas para a entrevista das enfermeiras, na tentativa de avaliar a adesão e a qualidade das consultas de pré-natal realizadas por esses profissionais. A entrevista foi realizada presencialmente em cada UBS, e apos concordância e assinatura do TCLE, foi entregue o questionário de forma impressa, onde a pesquisadora fez os questionamentos de forma oral e transcrita as respostas pelo profissional de saúde. A pesquisa foi realizada no período de agosto de 2021.

O questionário foi dividido em 3 etapas, a $1^{\circ}$ abordando questões sobre a identificação da UBS e identificação das enfermeiras, assim como nome, idade, tempo de formação e tempo de experiência na UBS que trabalha. A $2^{\circ}$ etapa, constituise de questões sobre a adesão das gestantes ao pré-natal, a $3^{\circ}$ e última aborda questões pertinentes a assistência ao pré-natal, como, ausculta de batimentos cardíacos fetais, medição da altura uterina, orientações sobre cuidados com o recém-nascido e a amamentação, solicitação e avaliação de exames laboratoriais, bem como, a prescrição da suplementação da mulher gravida. Para avaliar a estrutura física, foi observado de forma in loco pela pesquisadora, seguindo um formulário em check list adaptado pela mesma.

A tabulação de dados e gráficos foram realizados em planilha eletrônica do Microsoft excel@ e a redação de textos foi utilizado o programa Microsoft Office Word ®. Esta pesquisa foi aprovada pelo Comitê de ética em Pesquisa da Universidade UNICEUMA, sob o protocolo CAAE n. ${ }^{\circ} 44615221.0 .0000 .5084$.

\section{Resultados}

O estudo avaliou 4 questionários de profissionais da enfermagem que fazem parte da Estratégia Saúde da família do município de campestre do Maranhão e que realizam a consulta de pré-natal.

Dos 4 participantes, todas eram do sexo feminino. A faixa etária variou de 24 a 39 anos. Das enfermeiras, 1 (25\%) tem 39 anos, $1(25 \%) 34$ anos, $1(25 \%) 33$ anos e 1 (25\%) 24 anos. (Figura 1)

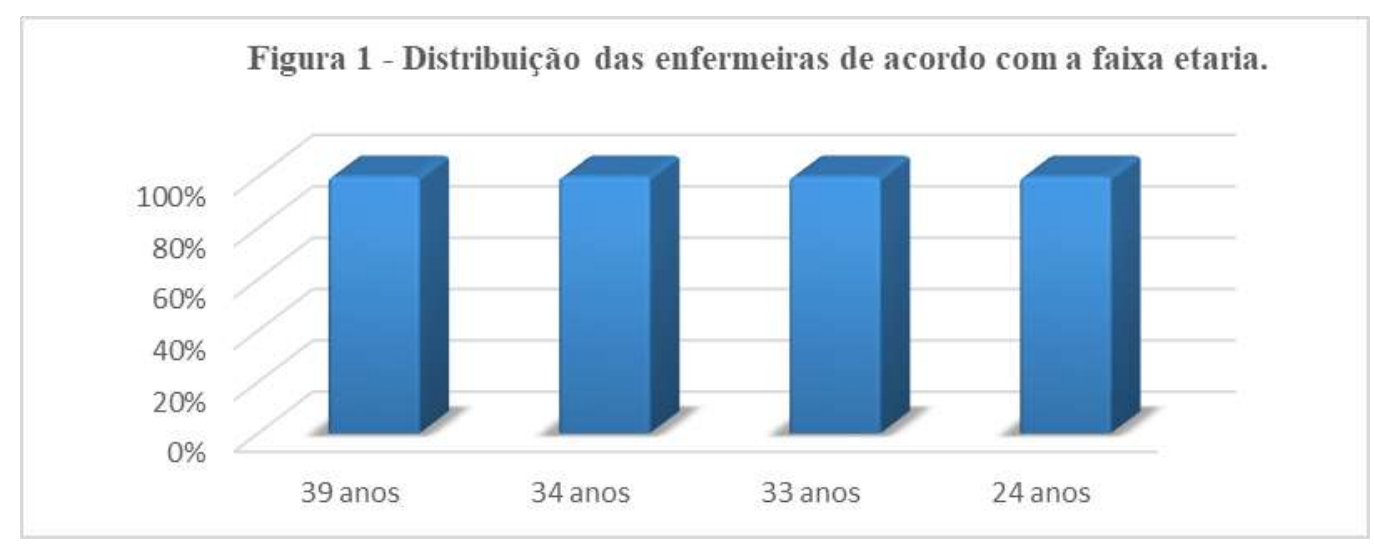

Fonte: Lima, Suelane Coelho (2021). 
Quanto ao tempo de formação, podemos observar que 50\% das entrevistadas possuem 8 anos de formação, $25 \%$ há 1 ano e $25 \%$ está formada há 6 meses. Desses profissionais, o tempo de atuação na UBS variou de 1 mês a 8 anos, sendo que $75 \%$ atuam há menos de 1 ano nas unidades, e somente 1 atua a 8 anos.

Quanto a adesão das gestantes ao pré-natal do município, 100\% afirmaram que a adesão e a busca de novas gestantes ao programa são realizadas pelos agentes comunitários de saúde, seguindo o protocolo recomendado pelo Ministério da Saúde, e por iniciativa própria da gestante ao procurar o atendimento. 100\% afirmou conseguir cobrir todas as gestantes das suas áreas. Em relação às gestantes faltosas, $100 \%$ afirma que essa busca é realizada pelo enfermeiro via ligações ou aplicativos de comunicação e através dos agentes comunitários de saúde durante as visitas domiciliares.

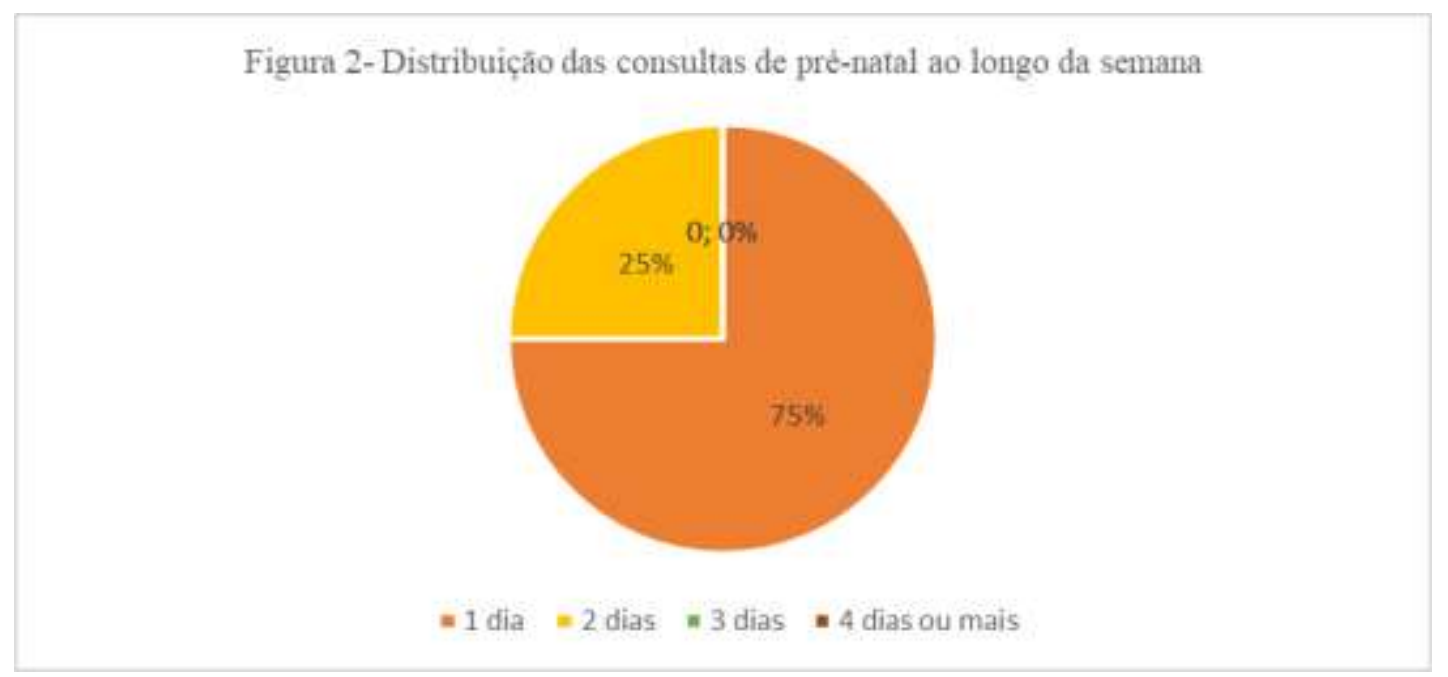

Fonte: Lima, S. C. (2021).

Sobre a organização do atendimento (Figura 2), 75\% das enfermeiras afirmaram separar 1 dia da semana para realizar a consulta de pré-natal e $25 \%$ afirmou separar 2 dias na semana devido à alta quantidade de gestantes da sua área, sobretudo, as mesmas evidenciaram que, a primeira consulta da gestante é realizada em todos os dias da semana assim que há procura do atendimento.

Quadro 1 - Profissionais que fazem parte do Pré-natal.

\begin{tabular}{|l|c|c|c|}
\hline Profissionais & Enf A & Enf B & Enf C \\
\hline Assistente Social & & $\mathrm{x}$ & $\mathrm{x}$ \\
\hline Enfermeiro & $\mathrm{x}$ & $\mathrm{x}$ & $\mathrm{x}$ \\
\hline Medico geral/ família & $\mathrm{x}$ & $\mathrm{x}$ & $\mathrm{x}$ \\
\hline Ginecologista/ obstetra & $\mathrm{x}$ & $\mathrm{x}$ & $\mathrm{x}$ \\
\hline Nutricionista & $\mathrm{x}$ & $\mathrm{x}$ & $\mathrm{x}$ \\
\hline Odontólogo & $\mathrm{x}$ & $\mathrm{x}$ & $\mathrm{x}$ \\
\hline Psicólogo & $\mathrm{x}$ & & $\mathrm{x}$ \\
\hline
\end{tabular}

Fonte: Lima, S. C. (2021).

Em relação aos profissionais de saúde que fazem parte do atendimento do pré-natal em algum momento (quadro 1), $50 \%$ afirmou que o Assistente Social faz parte da equipe multidisciplinar e são chamados ao atendimento caso haja necessidade. A enfermagem e medico geral e/ou médico da família conta com $100 \%$ de participação, sendo que, no município, 
as consultas de pré-natal são alternadas entre a enfermagem e os médicos. 75\% afirmou realizar um encaminhamento ao médico obstetra ou ginecologista caso haja necessidade do atendimento. 100\% relatou encaminhar as gestantes para avaliação com a nutricionista e dentistas, e 75\% afirmou que já precisou encaminhar para o serviço de psicologia e encaminha suas pacientes a depender da sua necessidade.

$100 \%$ dos profissionais afirmaram que todas as suas gestantes já saem da consulta com sua próxima consulta agendada, seja com a enfermeira ou com o médico. O município de Campestre não possui atendimento para as gestantes de alto risco, sendo assim, $100 \%$ das enfermeiras relataram que, todas as mulheres que se enquadram como gestante de alto risco e precisam de um acompanhamento maior, são encaminhadas para o serviço de referência do município, o Hospital Regional Materno Infantil do município de Imperatriz do Maranhão.

Quadro 2 - Aç̃̃es desenvolvidas na UBS para o cuidado das gestantes.

\begin{tabular}{|c|c|c|}
\hline Variáveis & $\mathbf{n}$ & $\%$ \\
\hline $\begin{array}{l}\text { Promoção, diagnóstico e tratamento de problemas clínicos em geral? } \\
\text { Sim }\end{array}$ & 4 & $100 \%$ \\
\hline $\begin{array}{l}\text { Promoção, diagnóstico e tratamento de problemas de saúde bucal? } \\
\text { Sim } \\
\text { Não }\end{array}$ & $\begin{array}{l}3 \\
1\end{array}$ & $\begin{array}{l}75 \% \\
25 \%\end{array}$ \\
\hline $\begin{array}{l}\text { Promoção, diagnóstico e tratamento de problemas de saúde mental? } \\
\text { Sim } \\
\text { Não }\end{array}$ & $\begin{array}{l}3 \\
1\end{array}$ & $\begin{array}{l}75 \% \\
25 \%\end{array}$ \\
\hline $\begin{array}{l}\text { Imunizações? } \\
\text { Sim }\end{array}$ & 4 & $100 \%$ \\
\hline $\begin{array}{l}\text { Planejamento familiar? } \\
\text { Sim }\end{array}$ & 4 & $100 \%$ \\
\hline $\begin{array}{l}\text { Promoção do aleitamento materno? } \\
\text { Sim }\end{array}$ & 4 & $100 \%$ \\
\hline $\begin{array}{l}\text { Promoção de hábitos alimentares saudáveis? } \\
\text { Sim }\end{array}$ & 4 & $100 \%$ \\
\hline $\begin{array}{l}\text { Promoção da atividade física? } \\
\text { Sim }\end{array}$ & 4 & $100 \%$ \\
\hline
\end{tabular}

Fonte: Lima, S. C. (2021).

Dos entrevistados, 100\% afirmaram realizar a promoção, diagnóstico e tratamento de problemas clínicos em geral nas gestantes, promoção da imunização, planejamento familiar, promoção do aleitamento materno, promoção de hábitos alimentares saudáveis e promoção da atividade física. (Quadro 2).

No entanto, desses enfermeiros, $25 \%$ afirmou não realizar a promoção, diagnóstico e tratamento de saúde bocal e saúde mental, enquanto os outros $75 \%$ afirmaram realizar. (Quadro 2). $100 \%$ afirmaram utilizar a caderneta da gestante em todas as consultas, assim como o preenchimento dela. 100\% relata a prescrição de Sulfato ferroso e Acido Fólico, suplementos utilizados durante o período gestacional, seguindo o protocolo estabelecido pelo ministério da saúde. $100 \%$ afirmam realizar a ausculta de batimentos cardíacos fetais (BCF) juntamente com a gestante durante a consulta. 100\% afirma realizar a medição da altura uterina (AU) em todas as consultas. 100\% afirmou fazer a solicitação de USG obstétrica durante a gestação assim que necessário e realizar busca por doenças pré-existentes.

$100 \%$ afirma orientar as gestantes sobre a alimentação durante todo o período da gestação, assim como o ganho de peso adequado para cada trimestre. 100\% relata observar a data da próxima vacina e chamar a atenção, caso esteja atrasada. 100\% afirma encaminhar suas gestantes para a avaliação da saúde bucal, orientar sobre a promoção do aleitamento materno de forma correta, orientar gestantes sobre os cuidados com o recém-nascido. 
Apenas 75\% afirma orientar a gestante sobre atividade física, riscos do tabagismo, álcool e outras drogas durante a gravidez. No entanto, $100 \%$ das enfermeiras, afirmam conversar e passar orientações sobre a anticoncepção no pós-parto. $100 \%$ afirmam realizar a revisão puerperal em até 7 dias do pós parto.

Quanto a duração do atendimento, $75 \%$ afirma que o atendimento demora de 30 minutos ou mais, enquanto $25 \%$ realiza a consulta entre 15 a 20 minutos. $50 \%$ das entrevistadas realizam grupos de gestantes.

Tabela 1 - Solicitação de exames laboratoriais.

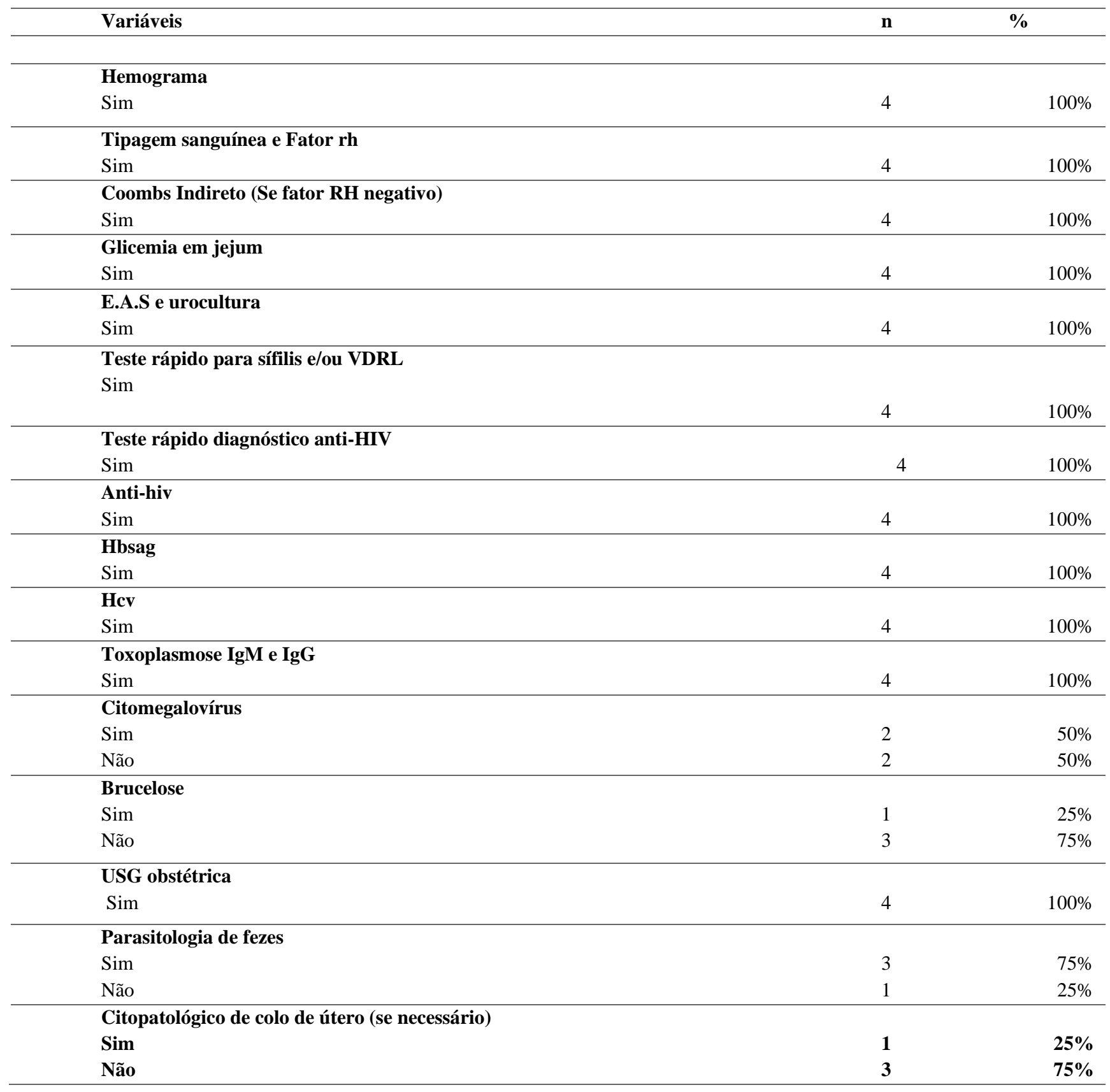

Fonte: Lima, S. C. (2021).

No que diz respeito a solicitação de exames laboratoriais, $100 \%$ das entrevistadas afirmaram solicitar os seguintes exames, hemograma, tipagem sanguínea e fator $\mathrm{rH}$, coombs indireto (se fator $\mathrm{rH}$ negativo), glicemia em jejum, EAS e urocultura, testes rápidos para sífilis e/ou VRDL, teste rápido de HIV e anti-hiv, hbsag, hcv, toxoplasmose IgG e IgM e USG 
obstétrica (Tabela 1). No entanto, 50\% afirmaram solicitar o exame de citomegalovírus, enquanto 25\% afirmou solicitar Brucelose, $75 \%$ solicitam parasitologia de fezes, enquanto $25 \%$ afirmou costumar solicitar citopatológico de colo de útero (se necessário) (Tabela 1).

$100 \%$ afirmaram utilizar a caderneta da gestante em todas as consultas, assim como o preenchimento dela. $100 \%$ relata a prescrição de Sulfato ferroso e Acido Fólico, suplementos utilizados durante o período gestacional, seguindo o protocolo estabelecido pelo ministério da saúde. 100\% afirmam realizar a ausculta de batimentos cardíacos fetais (BCF) juntamente com a gestante durante a consulta. $100 \%$ afirma realizar a medição da altura uterina (AU) em todas as consultas. 100\% afirmou fazer a solicitação de USG obstétrica durante a gestação assim que necessário e realizar busca por doenças préexistentes.

$100 \%$ afirma orientar as gestantes sobre a alimentação durante todo o período da gestação, assim como o ganho de peso adequado para cada trimestre. 100\% relata observar a data da próxima vacina e chamar a atenção, caso esteja atrasada. 100\% afirma encaminhar suas gestantes para a avaliação da saúde bucal, orientar sobre a promoção do aleitamento materno de forma correta, orientar gestantes sobre os cuidados com o recém-nascido.

Apenas $75 \%$ afirma orientar a gestante sobre atividade física, riscos do tabagismo, álcool e outras drogas durante a gravidez. No entanto, $100 \%$ das enfermeiras, afirmam conversar e passar orientações sobre a anticoncepção no pós-parto. 100\% afirmam realizar a revisão puerperal em até 7 dias do pós parto.

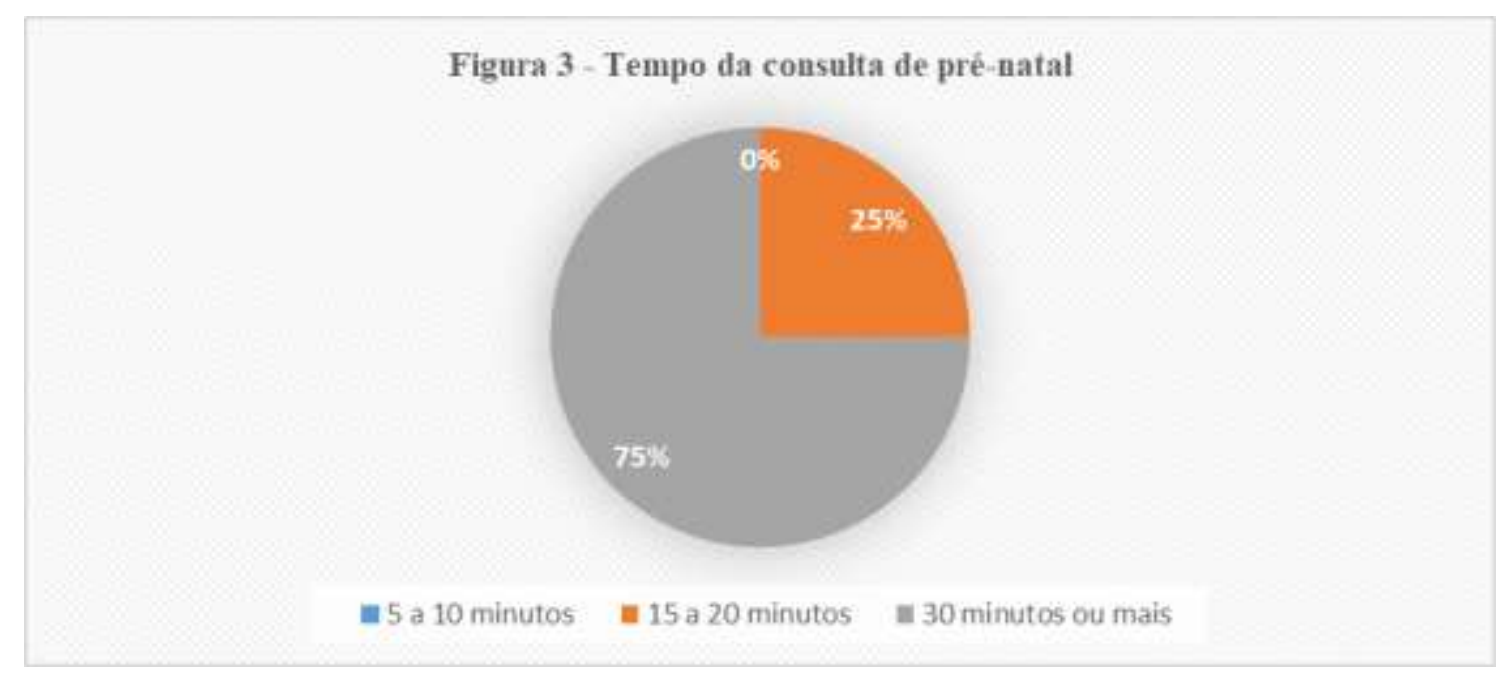

Fonte: Lima, S. C. (2021).

Quanto a duração do atendimento (Figura 3), 75\% afirma que o atendimento demora de 30 minutos ou mais, enquanto $25 \%$ realiza a consulta entre 15 a 20 minutos. $50 \%$ das entrevistadas realizam grupos de gestantes.

\section{Discussão}

Participou dessa pesquisa, 4 enfermeiras, todas do sexo feminino, a idade variou de 24 a 39 anos. Neste estudo não foi estabelecido relação entre idade e a atuação do enfermeiro nos atendimentos pré-natais. No que se refere a graduação, $50 \%$ das entrevistadas estão formadas há 8 anos, enquanto 50\% tem em média 1 ano de formação. Observou-se que, 75\% dos profissionais, estão há menos de 1 ano atuando nas unidades. E se falar em qualidade do pré-natal e melhoria do atendimento, priorizar um acompanhamento por um longo tempo é essencial. No entanto, vemos que na maioria das UBS os profissionais são contratados, o que dificulta este atendimento (Matuella, 2015).

No município, a adesão das gestantes ao pré-natal na maior parte se dá através de agentes comunitários de saúde, 
seguindo o protocolo do MS, no qual é atribuição do agente comunitário de saúde realizar visitas domiciliares para a identificação das gestantes e encaminhar essas gestantes para o serviço de saúde, visando a captação precoce para a adesão ao pré-natal (Brasil, 2012). No entanto, há mulheres em que procuram o atendimento por iniciativa própria. Em relação às gestantes faltosas, os agentes comunitários de saúde na maior parte das vezes vão em busca da gestante, através da busca ativa, orientando sobre a periodicidade das consultas (Brasil, 2012), as entrevistadas afirmaram ainda que, as mesmas também fazem busca ativa das gestantes faltosas através de ligações e aplicativos de mensagem, estando em contato direto com as mesmas.

Com relação aos profissionais que fazem parte da equipe multidisciplinar no atendimento, observou-se que todas as entrevistadas alegaram que fazem parte da equipe, o médico clinico geral/ da família, enfermeiro, nutricionista e cirurgiãodentista. Segundo o Manual de Atenção ao Pré-natal de baixo risco (Brasil, 2012), a equipe multidisciplinar é composta por agentes comunitários de saúde, técnico de enfermagem, enfermeiro, medico e cirurgião-dentista. Enquanto aos outros profissionais, 75\% dos entrevistados afirmaram o encaminhamento para um ginecologista/ obstetra e para a psicóloga, enquanto 50\% afirmou encaminhar para a assistência social, caso necessidade. Tratando-se da Rede Cegonha, o programa é composto pelos seguintes profissionais: enfermeiro, técnico de enfermagem, médico, cirurgião-dentista e, no caso das Estratégias Saúde da Família (ESF), o agente comunitário da saúde. No entanto, podem ser incluídos os seguintes profissionais, terapeutas ocupacionais, nutricionistas e fisioterapeutas (Sales et al., 2021). No município de Campestre-MA, as consultas são realizadas de forma alternada entre o enfermeiro e o médico. Seguindo o protocolo preconizado pelo Caderno de Atenção ao Pré-natal de baixo risco.

Em relação as ações desenvolvidas na UBS para o cuidado com as gestantes, todas as enfermeiras afirmaram realizar a promoção, diagnóstico e tratamento de problemas clínicos em geral nas gestantes, promoção da imunização, planejamento familiar, promoção do aleitamento materno, promoção de hábitos alimentares saudáveis e promoção da atividade física. No entanto, desses enfermeiros, $25 \%$ afirmou não realizar a promoção, diagnóstico e tratamento de saúde bocal e saúde mental, enquanto os outros 75\% afirmaram realizar. Segundo Dias et al. (2015) as gestantes têm a consciência de que é importante entender sobre assuntos durante o seu pré-natal, de modo que este conhecimento assegure uma gestação, parto e puerpério sem complicações.

De acordo com Carrara e Oliveira (2013), a gestante se sente satisfeita através da qualidade do atendimento prestado pelo enfermeiro, ao esclarecer suas dúvidas, orientações que serão primordiais para o cuidado do recém-nascido.

Sobre o registro na caderneta das gestantes, $100 \%$ afirmou solicitar a carteira, assim como o preenchimento adequado da mesma. O registro dos atendimentos na caderneta da gestante é importante para a assistência ao pré-natal nos serviços de saúde, para que o cuidado seja de forma contínua, com o histórico completo da gestação, assistência ao parto, promovendo assim a tomada de decisões da melhor forma diante de uma situação adversa (Sales; Semente; Fernandes, 2013).

$100 \%$ afirmou prescrever a suplementação de Sulfato ferroso e ácido fólico, além de orientar para a automedicação, seguindo a risca a prescrição. Cabe ao enfermeiro a prescrição de suplementos de acordo com o protocolo do ministério da saúde e orientar a ingestão de forma correta. O Ministério da Saúde recomenda a suplementação de Ácido fólico para prevenir defeitos no tubo neural do bebê, podendo ser usado 2 meses antes da gestação e nos 2 primeiros meses de gestação. Enquanto a ingestão de Sulfato ferroso, vá prevenir os baixos níveis de hemoglobina no parto e puerpério (Saúde, 2012).

Em relação a ausculta do BCF e medida da altura uterina, todas as enfermeiras afirmaram realizar durante suas consultas juntamente com as gestantes. A ausculta dos batimentos cardíacos fetais deve ser realizado com o sonar, após 12 semanas, visando constatar a presença, ritmo, frequência e a normalidade desses batimentos. Enquanto a medida da altura uterina, deve ser realizada no intuito de identificar o crescimento fetal e diagnosticar qualquer anomalia a partir da relação entre a autura uterina e a idade gestacional (Baptista et al., 2015). No que diz respeito a orientação sobre o aleitamento materno, todas as enfermeiras afirmaram orientar as gestantes sobre a forma correta, bem como a pegada e suç̧ão correta. De 
acordo com o protocolo ministerial, as orientações para o aleitamento materno devem ser feitas em diferentes momentos de forma educativa, visto que evita diarreia, infecção respiratória, diminui o risco de alergias, colesterol alto, diabetes, hipertensão, reduz os riscos de obesidade bem como prevenir o risco de mortes infantis.

Ações como, imunizações, orientações sobre alimentação, ganho de peso, encaminhamento para avaliação da saúde bucal, orientações sobre os cuidados com o recém-nascido, são da responsabilidade do enfermeiro. Todas as enfermeiras afirmam realizar essas ações durante seus atendimentos. No entanto, apenas 3 afirmam orientar sobre os riscos do álcool, drogas e tabagismo durante a gestação, assim como a prática de atividade física.

De acordo com Grinfeld (2009) o consumo excessivo de álcool durante a gravidez é um dos problemas mais relevantes da dependência alcoólica, pois pode ocasionar a síndrome alcoólica fetal (SAF), ou seja, um comprometimento neuropsiquiátrico em filhos de mulheres que beberam em excesso durante a gestação.

Quanto a anticoncepção pós parto, as 4 enfermeiras afirmaram orientar suas pacientes sobre a anticoncepção, o uso e o risco de não usar após o período puerperal. Em relação à revisão puerperal, segundo as entrevistadas, todas conseguem realizar a revisão puerperal ainda na primeira semana pós parto. Segundo o Manual técnico de pré-natal e puerpério (2006), a atenção a mulher e ao recém-nascido no pós parto imediato e nas primeiras semanas é fundamental para a saúde materna e neonatal. Recomenda-se que a visita domiciliar na primeira semana após a alta do bebe.

Para Vieira et al. (2008), medidas como o planejamento familiar representa uma ação de extrema importância para a preservação do bem-estar materno-fetal, prevenindo assim uma possível gestação não planejada e suas consequências.

Em relação ao tempo de consulta, $75 \%$ afirmam que sua consulta demora 30 minutos ou mais, enquanto $25 \%$ afirma que suas consultas têm um tempo de 15 a 20 minutos. 50\% afirma ainda que fazem grupos de encontro com gestantes. As reuniões educativas com as gestantes possibilitam ao enfermeiro transmitir o conhecimento acerca do processo gestacional e utilizar essa ferramenta como meio de promoção a saúde (Marcon; Roecker; Souza, 2010).

No que diz respeito a solicitação dos exames laboratoriais, todas as entrevistadas afirmaram solicitar os seguintes exames, hemograma, tipagem sanguínea e fator $\mathrm{rH}$, coombs indireto (se fator $\mathrm{rH}$ negativo), glicemia em jejum, EAS e urocultura, testes rápidos para sífilis e/ou VRDL, teste rápido de HIV e anti-hiv, sorologia para hepatite B, sorologia para hepatite C, toxoplasmose IgG e IgM e USG obstétrica. No entanto, 2 afirmaram solicitar o exame de citomegalovírus, enquanto 1 afirmou solicitar Brucelose, 3 solicitam parasitologia de fezes, enquanto 1 afirmou costumar solicitar citopatológico de colo de útero (se necessário). Os exames são solicitados em trimestres diferentes de acordo com a idade gestacional, no primeiro trimestre são solicitados todos os exames recomendados; no segundo trimestre, coombs indireto é solicitado, se a mãe for Rh negativa e o teste de tolerância a glicose 75\%; no terceiro trimestre, alguns testes do primeiro são repetidos (Leal; Barreiro; Mendes, 2018).

Os exames laboratoriais de rotina no pré-natal são uteis para identificar precocemente uma doença gestacional, tratar, prevenir infecções fetais e até óbito materno e infantil. Com os resultados dos exames laboratoriais, possibilita realizar e monitorar a classificação de risco gestacional (Paris; Pelloso; Martins, 2013).

De acordo com Brasil, a ultrassonografia pode ser solicitada durante a rotina quando houver disponibilidade, em caso de impossibilidade da determinação da idade gestacional na consulta, deve ser solicitado o mais precocemente possível. Segundo Baptista (2015) A não solicitação da ultrassonografia durante a gestação não constitui falha, nem diminui a qualidade do pré-natal. No entanto, é justificado a realização do exame no primeiro trimestre para determinar a medida de transluscência nucal, com o objetivo de rastrear anomalias cromossômicas, além de confirmar a presença dos batimentos cardíacos fetais.

Sobre a avaliação da estrutura física e equipamentos, feito de modo in loco, seguindo um formulário em check list. Identificou que os profissionais possuem todos os materiais, equipamentos para exames dentro da sala de consultório, como, mesas e cadeiras para entrevista, mesa de exame ginecológico, foco de luz, balança para adultos, esfigmomanômetro e 
estetoscópio clinico, sonar Doppler e gel lubrificante, fita métrica, cadernos e impressos para controle das gestantes, fichas para requisição de exames e prescrições de medicamentos, luvas e cartão das gestantes. No que se refere a estrutura física, a portaria 2.226/2009 do Ministério da saúde, estabelece a estrutura física mínima para as unidades básicas de saúde, deve conter: sala de espera e recepção, consultório, consultório odontológico, sala de procedimentos e curativo, sala exclusiva de vacinas, sala de reuniões, copa/cozinha, área de depósito de materiais de limpeza, banheiros para a população e funcionários (Brasil, 2009).

\section{Considerações Finais}

No município de Campestre, as Unidades Básicas de saúde constituem-se como porta de entrada para a realização do pré-natal, sendo, o enfermeiro o primeiro profissional a ter um contato direto com a gestante. Para isso, os enfermeiros da atenção primariam devem estar preparados para assumir essa posição, bem como, estar preparado para implementar medidas e ações no cuidado da gestante. O enfermeiro deve estar atento para dúvidas e anseios biopsicossocial da mulher gravida bem como da sua família.

Através deste estudo, conseguimos concluir que a equipe de enfermagem do município de Campestre, desempenha um papel importante na realização do pré-natal. Com a análise dos dados obtidos, podemos observar que o pré-natal deve ser feito de modo a acolher a gestante, de forma humanizada, qualificada.

Os resultados evidenciam que a qualidade das ações esperadas na assistência, preconizadas pelo Caderno da atenção ao pré-natal de baixo risco, foram desenvolvidas de modo satisfatório, preenchendo e cumprindo os requisitos para um atendimento de qualidade. Observou-se que a assistência prestada, visto que, além dos procedimentos e exames de rotina são realizados, bem como os exames complementares.

Apesar da falta de ações em grupos, da educação continua para com a gestantes, sugere-se a partir deste estudo, que seja feita mais capacitações para os profissionais de saúde das UBS, sobre a temática. Faz-se necessário, o investimento na qualificação profissional para o atendimento de todo o ciclo gravídico.

É necessário, que as enfermeiras orientem suas gestantes sobre os riscos do consumo de álcool e drogas na gestação, orientando, alertando e sanando as dúvidas sobre as complicações decorrentes de abuso desse tipo de substâncias. Apesar das dificuldades encontradas, os objetivos propostos pelo estudo foram alcançados, avaliando as ações da enfermagem na assistência. Espera-se que este estudo leve uma reflexão crítica para as enfermeiras e gestores do município, a fim de aprimorar a assistência.

Neste sentido, faz-se necessários mais estudos a respeito do assunto, no sentido de contribuir para a melhoria da qualidade da assistência prestada pelos enfermeiros.

\section{Referências}

Baptista, R. S., Dutra, M. O. M., Coura, A. S., \& de Sousa, F. S. (2015). Assistência pré-natal: ações essenciais desenvolvidas pelos enfermeiros. Enfermería Global, 14(4), 96-142.

Barbosa, M. A. (2007). Avaliação da assistência pré-natal de baixo risco no Município de Francisco Morato.

Brasil. (2002). Programa de humanização do parto: Humanização no pré-natal e nascimento. Ministério da Saúde.

Brasil. (2005). Pré-natal e Puerpério: atenção qualificada e humanizada - manual técnico. Ministério da Saúde.

Brasil. (2009). Portaria n 2.226, de 18 de setembro de 2009. Institui, no âmbito da Política Nacional de Atenção Básica, o Plano Nacional de Implantação de Unidades Básicas de Saúde para Equipes de Saúde da Família. Diário Oficial da União.

Brasil. (2012). Atenção ao pré-natal de baixo risco. Cadernos de Atenção Básica, n 32. Ministério da Saúde. 
Cei, N. V. S., Sales, A. P. M., Pereira, M. R. L., de Carvalho, E. C., de Souza Mascarenhas, L. R., \& do Vale Silva, T. B. (2019). Rede Cegonha e equipe multiprofissional no pré-natal e puerpério de Unidades Básicas de Saúde de Belém, Pará. Pará Research Medical Journal, 3(1), 0-0.

de Andrade, F. M., de Lima Castro, J. F., \& da Silva, A. V. (2016). Percepção das gestantes sobre as consultas médicas e de enfermagem no pré-natal de baixo risco. Revista de Enfermagem do Centro-Oeste Mineiro, 6(3).

de Brito, G. V., Albuquerque, I. M. A. N., Ribeiro, M. A., Ponte, E. C. S., Moreira, R. M. M., \& Linhares, M. D. G. C. (2018). Consulta de puericultura na estratégia saúde da família: Percepção de enfermeiros. Revista de APS, 21(1).

de Sousa, L. T., de Luna Benicio, A., \& de Santana, M. D. R. (2017). Percepção de enfermeiros da estratégia saúde da família em relação ao pré-natal com adolescentes. SANARE-Revista de Políticas Públicas, 16(1).

dos Santos Viana, C., de Almeida, D. R., de Campos, A. L., de Almeida Silva, A., de Oliveira, C. S., da Silva Dias, N., \& dos Santos, T. M. (2021). Qualidade da assistência pré-natal realizada por enfermeiros das unidades básicas de saúde de Cáceres-MT. Brazilian Journal of Health Review, 4(1), $3173-3193$.

Dias, E. G., dos Anjos, G. B., Alves, L., Pereira, S. N., \& Campos, L. M. (2018). Ações do enfermeiro no pré-natal e a importância atribuída pelas gestantes. Revista Sustinere, 6(1), 52-62.

Gil, A. C. (2002). Como elaborar projetos de pesquisa (Vol. 4, p. 175). Atlas.

Grinfeld, H. (2009). Consumo nocivo de álcool durante a gravidez. Álcool e suas consequências: uma abordagem multiconceitual. São Paulo: Manole, 8(3), 179-99.

Leal, N. D. J., Barreiro, M. D. S. C., Mendes, R. B., \& Freitas, C. K. A. C. (2018). Assistência ao pré-natal: depoimento de enfermeiras. Rev. pesqui. cuid. fundam. (Online), 113-122.

Lemes, A. G. (2012). Assistência de enfermagem a gestante na primeira consulta de pré-natal. Revista Eletrônica Interdisciplinar, 2(8).

Matuella, M. (2019). Melhoria no Programa de Pré-Natal e Puerpério na Unidade Básica de Saúde Esplanada de Caxias do Sul, RS.

Paris, G. F., Pelloso, S. M., \& Martins, P. M. (2013). Qualidade da assistência pré-natal nos serviços públicos e privados. Revista Brasileira de Ginecologia e Obstetrícia, 35, 447-452.

Queiroz, D. J. M., Soares, D. B., \& de Oliveira, K. C. A. N. (2015). Avaliação da assistência pré-natal: relevância dos exames laboratoriais. Revista Brasileira em Promoção da Saúde, 28(4), 504-512.

Sales, K. C., SEMENTE, P., \& Fernandes, E. R. L. (2013). Cartão da Gestante: um elo fundamental. Revista Brasileira de Informações Científicas, 4(3), 8-15. Silva, D. A. D. (2020). Cuidado ao pré-natal segundo indicadores do programa de humanização do pré-natal e nascimento. Rev. enferm. atenção saúde, 111123.

Souza, V. B., Roecker, S. I. M. O. N. E., \& Marcon, S. S. Educação em Saúde na Assistência Pré-Natal: Percepção de gestantes atendidas na rede básica de Maringá-PR. XlX Jornada de Cascavelense de Enfermagem, 10-12.

Valente, M. M. Q. P., de Freitas, N. Q., Áfio, A. C. E., de Sousa, C. S. P., Evangelista, D. R., \& Moura, E. R. F. (2013). Assistência pré-natal: um olhar sobre a qualidade. Rev Rene, 14(2), 280-289.

Vieira, C. S., Brito, M. B., \& Yazlle, M. E. H. D. (2008). Contracepção no puerpério. Revista Brasileira de Ginecologia e Obstetrícia, $30,470-479$. 